\title{
Análise de Cluster e Análise Heurística na construção de uma tipologia de alcoolistas
}

\author{
Cluster Analysis and Heuristic Analysis in building a typology of alcoholics \\ Mário Sérgio Ribeiro', Luiz Cláudio Ribeiro², Rafael Augusto Ferreira³
}

\section{RESUMO}

Objetivos: Desenvolver e avaliar uma tipologia binária de alcoolistas. Métodos: Analisados registros de 329 alcoolistas acompanhados em estudo prospectivo e observacional. Análise de Correspondência foi aplicada para selecionar o menor número de variáveis capazes de representar a máxima variabilidade dos sujeitos; Análise de Cluster para identificar dois subtipos; e Análise Heurística para organizar as variáveis selecionadas em grupos de sentido. Análises Bivariadas foram utilizadas para testar possíveis associações com 51 variáveis demográficas ou clinicamente relevantes. Resultados: A Análise de Correspondência selecionou 20 variáveis, posteriormente categorizadas em três grupos de sentidos por meio de Análise Heurística: "comportamentos", "crenças" e "sentimentos". Os pacientes do subgrupo

\section{Palavras-chave}

Alcoolismo, tipologia, diagnóstico, análise por conglomerados, análise heurística.

\section{Keywords}

Alcoholism, typology, diagnosis, cluster analysis, heuristic analysis. 1 possuem perfil de maior gravidade clínica, psicopatológica e social. Sentimentos e crenças identificados se associaram a variáveis de consumo e recaída e os comportamentos com repercussão social. Conclusões: Os resultados deste estudo permitem supor que a tipologia aqui apresentada possa contribuir para o tratamento desses subtipos de alcoolistas identificados por meio de estratégias teórico-práticas adaptadas às necessidades desses sujeitos.

\begin{abstract}
Objectives: To develop and to describe a binary typology of alcoholics. Methods: Data derived from 329 patients followed in an observational study. Correspondence Analysis was applied to identify the least number of variables capable of representing the maximum variability of the subjects; Cluster Analysis was performed to identify two subgroups; and Heuristic Analysis performed with the objective of organizing the variables into groups of meaning. Bivariate Analyses were used to test possible associations of Clusters to 51 variables - demographic or of clinical relevance. Results: Correspondence Analysis derived 20 variables, afterwards categorized into three groups of meaning through Heuristic Analysis: "behaviors", "beliefs" and "feelings". The results indicate that subgroup 1 patients present a more severe clinical, psychopathological and social profile. Identified feelings and beliefs were associated to drinking and relapsing variables, and behaviors to social repercussions. Conclusions: The results of this study suggest that the typology here presented may contribute to the treatment of these alcoholics subtypes by means of therapeutic strategies adapted to the identified characteristics of each subgroup of patients.
\end{abstract}

1 Universidade Federal de Juiz de Fora (UFJF), Faculdade de Medicina; Laboratório de Pesquisas em Personalidade, Álcool e Drogas (Lappda-UFJF), Filosofia.

2 UFJF, Departamento de Estatística; Laboratório de Estudos Estatísticos na Saúde (LEES-UFJF), Demografia. 3 UFJF, Medicina.

Endereço para correspondência: Mário Sérgio Ribeiro Rua Severino Meireles, 325/902 36025-040 - Juiz de Fora, MG, Brasil

E-mail: mariosribeiro@acessa.com 


\section{INTRODUÇÃO}

Tipologias representam sistemas de classificação e conjuntos de definições utilizados para diferenciar subtipos relativamente homogêneos de uma população'. Assim como em outras patologias, as classificações tipológicas em alcoolismo objetivam otimizar o diagnóstico, sugerir o prognóstico, medidas preventivas e/ou direcionar o tratamento por meio de esquemas classificatórios simples e práticos ${ }^{2,3}$.

Estudos mais antigos sobre tipologias de alcoolistas já forneceram evidências suficientes para comprovar a heterogeneidade clínica e etiológica desse grupo de sujeitos ${ }^{4}$. As principais tipologias de alcoolismo desenvolvidas ao longo do século XX indicam a presença de dois tipos básicos de alcoolistas, mais frequentemente encontrados e descritos pelos autores 5 .

Na prática, a maioria das tipologias de alcoolismo se situa entre abordagens exclusivamente clínicas e experimentais, utilizando, assim, um modelo misto ${ }^{6}$. Tais tipologias têm procurado construir uma ponte entre rigor científico e utilidade clínica, possibilitando a definição de grupos que respondam diferenciadamente a abordagens terapêuticas específicas ${ }^{5}$. Todavia, apesar das muitas similaridades entre as tipologias existentes, ainda não se chegou a uma classificação que preencha critérios ideais ${ }^{7}$. Ainda que se aponte para a possibilidade de que o aumento do número de subtipos possa contribuir para a construção de modelos clinicamente mais efetivos, essa linha de pesquisa é mais afeita aos aspectos neurobiológicos do alcoolismo do que aos domínios e abordagens psicossociais ${ }^{8-10}$.

Uma relevante dificuldade na abordagem do alcoolismo reside em sua origem multifatorial, resultante da interação de fatores genéticos, psicológicos, comportamentais e socioculturais ${ }^{11}$. A abordagem multidimensional passou a ser mais caracteristicamente adotada na segunda metade do século passado e vários autores propuseram tipologias multidimensionais de alcoolistas. As tipologias de alcoolistas mais estudadas nas últimas décadas são as de Cloninger e de Babor, que descrevem dois subtipos básicos ${ }^{5}$.

De acordo com Del Boca' ${ }^{12}$, apesar dos avanços na compreensão da multidimensionalidade do alcoolismo, pesquisas em tipologia do alcoolismo deveriam trabalhar com dados coletados tendo a classificação tipológica como finalidade. Para além de discutir as limitações de técnicas exclusivamente estatísticas para o desenvolvimento de subtipos, os pesquisadores deveriam expandir o uso de abordagens teóricas alternativas para explicar a estrutura dos dados e examinar o processo de constituição desses subtipos e suas possibilidades preditivas ${ }^{12,13}$. Babor e Caetano ${ }^{7}$ reforçam ainda a importância de que uma formulação tipológica possa contribuir para o pareamento entre paciente e serviço, ou modalidade terapêutica. Apontando para a questão da efetividade dos serviços, eles insistem que, idealmente, uma tipologia deveria ser adequada e viável aos serviços e seus clientes.
Segundo Leggio et al. ${ }^{8}$, novas pesquisas tipológicas deveriam se empenhar em explorar a possível aplicação de classificações tipológicas na prática clínica e direcionar-se para padronizar as pesquisas objetivando a clarificação das diferenças e das consistências compartilhadas pelas tipologias. Outra lacuna de provável relevância para a abordagem terapêutica de sujeitos com uso disfuncional de alcoólicos diz respeito aos aspectos psicossociais passíveis de serem abrangidos por novas tipologias.

O objetivo deste estudo foi aprofundar o estudo de um amplo banco de dados - resultante do processo de avaliação de pacientes de um serviço ambulatorial de referência para alcoolistas (PADQ), mas especificamente desenhado para objetivos de pesquisa - visando identificar o menor número de variáveis capazes de representar as características dos alcoolistas estudados e, em seguida, desenvolver uma tipologia binária, passível de ser diretamente avaliada diante de tipologias mais comumente descritas e estudadas, nomeadamente as tipologias de Cloninger e de Babor.

\section{MÉTODOS}

Para a elaboração deste artigo, partiu-se de um estudo observacional prospectivo - sem intervenção do grupo de pesquisadores na alocação dos sujeitos ou procedimentos terapêuticos, e aprovado pelos Comitês de Ética em Pesquisa (CEP) do HU-UFJF (Protocolo 099-23/2000-Grupo III) e CEP da UFJF (Protocolo CEP-UFJF 1071.117.2007) - realizado com pacientes adultos com transtornos mentais e do comportamento associados ao uso de alcoólicos e que tiveram seu primeiro atendimento no Programa de Atenção a Dependentes Químicos (PADQ) entre outubro de 1997 e dezembro de 2005. Foram incluídos neste estudo todos os 329 pacientes que tiveram a avaliação devidamente concluída pela equipe do PADQ.

A rotina de avaliação e de assistência do PADQ já foi apresentada e discutida anteriormente ${ }^{2,3}$. O processo de avaliação dos pacientes era realizado pelos técnicos responsáveis pela assistência, que registravam os dados em instrumentos estruturados e semiestruturados, alguns dos quais desenvolvidos com base em evidências empíricas de aspectos clínicos de relevância para a caracterização de alcoolistas'. Além da utilização do Inventário Multifásico Minnesota de Personalidade (MMP|14), a avaliação completa englobava os seguintes domínios: 1) Sociodemográfico; 2) Identificação do fator etiológico/padrão de consumo; 3) Identificação de fenômenos reforçadores e/ou desencadeadores do consumo; 4) Atitude do paciente ante sua problemática; 5) Identificação de complicações somáticas e psíquicas; 6) Exame físico; 7) Avaliação psicopatológica; 8) Diagnóstico principal e de comórbidos; 9) Delimitação da repercussão macro e microssocial.

Todas as variáveis aqui utilizadas decorrem diretamente dos prontuários e instrumentos de avaliação do serviço ou 
foram geradas por combinações dos registros clínicos. Para a realização das análises estatísticas, algumas variáveis - que, em sua ampla maioria, seguem o padrão de resposta "sim" ou "não" - com mais de duas possibilidades de resposta foram transformadas em dicotômicas, agrupando-se as categorias da forma mais lógica ou homogênea possível, conforme apresentado no quadro 1. Variáveis contínuas foram codificadas pela média do grupo - idade e número de doses de alcoólicos ingeridas semanalmente; os resultados das escalas do MMPI foram dicotomizados em acima e abaixo dos pontos de corte indicados na literatura ${ }^{14}$.

Todo o tratamento estatístico foi realizado com o uso do software SPSS for Windows 14.0 (licença 9656438). Como a hipótese multifatorial envolve o trabalho com diferentes dimensões e distintas perspectivas teóricas e a interação de um grande número de variáveis a elas vinculadas, foi inicialmente empreendida uma Análise de Correspondência (ACor) - uma ferramenta estatística que possibilita o tratamento concomitante de um grande número de variáveis com a finalidade de identificar um número mínimo de variáveis que expliquem a máxima variabilidade dessa população ${ }^{15}$.

Tomando-se as tipologias de Cloninger (16 variáveis) e de Babor (17 variáveis) como referência, o procedimento de ACor foi executado sobre as variáveis em formato dicotômico com o objetivo de se atingir de 15 a 20 variáveis representativas da variabilidade dos sujeitos. Com as variáveis selecionadas pela ACor, procedeu-se à Análise de Cluster, pelo método K-means, opção listwise, com solução em dois clusters.

Uma Análise Heurística ${ }^{16}$ - relativa aos conteúdos semânticos das variáveis responsáveis pela classificação dos indivíduos estudados nos dois clusters - foi então realizada, com o objetivo de organizar as variáveis por grupos de sentido.

Finalmente, análises bivariadas foram utilizadas para testar a significância das associações dos clusters com 16 variáveis sociodemográficas e outras 35 variáveis escolhidas por serem consideradas como clinicamente relevantes para a avaliação de alcoolistas'. A significância estatística foi verificada por meio do teste do Qui-quadrado de Pearson.

\section{RESULTADOS}

Os aspectos demográficos e socioeconômicos dos sujeitos incluídos e excluídos da análise estão apresentados no quadro 2. Ainda que para o conjunto de pacientes que não completou a fase de avaliações do PADQ tenha-se observado (dados não apresentados) mais elevado percentual de missing (não resposta) nas diversas variáveis, a avaliação dos dois grupos de pacientes praticamente não revelou diferenças entre eles. Apenas duas características foram significantemente associadas à maior conclusão da avaliação: não haver nascido em Juiz de Fora $(p=0,015)$ e ter idade acima da média do grupo $(p=0,004)$. Os pacientes incluídos na análise tinham, em média, 42,7 anos ( \pm 8,7 de desvio-padrão; mínima de 19 e máxima de 69 anos).

Quadro 1. Recodificação das variáveis apresentadas

\begin{tabular}{|c|c|c|}
\hline Variável & Categorias de resposta & Reagrupamentos \\
\hline \multirow{2}{*}{$\begin{array}{l}\text { Envolver-se em agressões } \\
\text { verbais }\end{array}$} & 1. Não & 1. Não \\
\hline & $\begin{array}{l}\text { 2. Raramente } \\
\text { 3. Frequentemente } \\
\text { 4. Sempre }\end{array}$ & 2. Sim \\
\hline \multirow[t]{2}{*}{ Uso de drogas ilícitas } & 1. Nenhuma & 1. Não \\
\hline & $\begin{array}{l}\text { 2. Cocaína } \\
\text { 3. Maconha } \\
\text { 4. Hipnóticos } \\
\text { 5. Solventes } \\
\text { 6. Opioides } \\
\text { 7. Alucinógenos } \\
\text { 8. Estimulantes }\end{array}$ & 2. Sim \\
\hline \multirow[t]{2}{*}{ Escolaridade } & $\begin{array}{l}\text { 1. Analfabeto } \\
\text { 2. Primeiro grau incompleto } \\
\text { 3. Primeiro grau completo }\end{array}$ & Baixa escolaridade \\
\hline & $\begin{array}{l}\text { 4. Segundo grau incompleto } \\
\text { 5. Segundo grau completo } \\
\text { 6. Terceiro grau incompleto } \\
\text { 7. Terceiro grau completo } \\
\text { 8. Pós-graduado }\end{array}$ & Maior escolaridade \\
\hline \multirow[t]{2}{*}{ Renda pessoal } & $\begin{array}{l}\text { 1. Nenhuma } \\
\text { 2. Menor que } 1 \mathrm{SM}^{*} \\
\text { 3.1 SM }\end{array}$ & Baixa renda \\
\hline & $\begin{array}{l}\text { 4. Entre } 1 \text { e } 3 \text { SM } \\
\text { 5. Entre } 3 \text { e } 6 \text { SM } \\
\text { 6. Entre } 6 \text { e } 10 \text { SM } \\
\text { 7. Entre } 10 \text { e } 20 \text { SM } \\
\text { 8. Mais de } 20 \text { SM }\end{array}$ & Moderada a alta renda \\
\hline \multirow[t]{2}{*}{ Renda familiar } & $\begin{array}{l}\text { 1. Menor que } 1 \text { SM } \\
\text { 2. } 1 \text { SM } \\
\text { 3. Entre } 1 \text { e } 35 M\end{array}$ & Baixa renda \\
\hline & $\begin{array}{l}\text { 4. Entre } 3 \text { e } 6 \text { SM } \\
\text { 5. Entre } 6 \text { e } 10 S M \\
\text { 6. Entre } 10 \text { e } 20 \text { SM } \\
\text { 7. Mais de } 20 \text { SM }\end{array}$ & Moderada a alta renda \\
\hline \multirow[t]{2}{*}{ Beber já causou problemas } & 1. Ninguém & 1. Não \\
\hline & $\begin{array}{l}\text { 2. Com cônjuge } \\
\text { 3. Com família } \\
\text { 4. Com vizinhos } \\
\text { 5. Com amigos } \\
\text { 6. Com desconhecidos } \\
\text { 7. No trabalho } \\
\text { 8. De doença } \\
\text { 9. De acidente } \\
\text { 10. Com polícia } \\
\text { 11. Com justiça }\end{array}$ & 2. Sim \\
\hline \multirow[t]{2}{*}{ Tem tido problemas sexuais } & 1. Não & 1. Não \\
\hline & $\begin{array}{l}\text { 2. Raramente } \\
\text { 3. Frequentemente } \\
\text { 4. Sempre }\end{array}$ & 2. Sim \\
\hline \multirow[t]{2}{*}{ Situação conjugal } & $\begin{array}{l}\text { 1. Solteiro } \\
\text { 2. Divorciado } \\
\text { 3. Viúvo }\end{array}$ & $\begin{array}{l}\text { 1. Sem relação conjugal } \\
\text { estável }\end{array}$ \\
\hline & $\begin{array}{l}\text { 4. Casado } \\
\text { 5. Amasiado }\end{array}$ & $\begin{array}{l}\text { 2. Vive em relação conjugal } \\
\text { estável }\end{array}$ \\
\hline
\end{tabular}

* SM: salário-mínimo. 
Quadro 2. Variáveis dos sujeitos que concluíram e que não concluíram a avaliação

\begin{tabular}{|c|c|c|c|}
\hline \multirow[b]{2}{*}{ Caracteristicas } & \multicolumn{2}{|c|}{ Sujeitos } & \multirow[b]{2}{*}{ p-valor" } \\
\hline & $\begin{array}{l}\text { Concluíram a } \\
\text { avaliação (\%) }\end{array}$ & $\begin{array}{l}\text { Não concluíram a } \\
\text { avaliaçăa (\%) }\end{array}$ & \\
\hline Sexo masculino & 88,1 & 90,1 & 0,443 \\
\hline Cor branca & 68,3 & 62,0 & 0,177 \\
\hline Idade acima da média do grupo & 57,4 & 44,1 & 0,004 \\
\hline Não nascidos em Juiz de Fora & 55,3 & 43,3 & 0,015 \\
\hline Baixa escolaridade* & 80,9 & 87,7 & 0,060 \\
\hline Sem relação conjugal estável & 50,2 & 51,5 & 0,780 \\
\hline Se casado, é o primeiro & 71,4 & 67,0 & 0,462 \\
\hline Tem filhos & 75,5 & 75,5 & 0,991 \\
\hline Tem até 2 filhos & 61,0 & 63,7 & 0,613 \\
\hline Tem até 4 moradores na residência & 67,7 & 65,1 & 0,575 \\
\hline Religião católica & 78,9 & 73,4 & 0,190 \\
\hline Frequenta 0 culto & 60,9 & 54,8 & 0,230 \\
\hline Não trabalham na profissão & 58,9 & 56,4 & 0,612 \\
\hline $\begin{array}{l}\text { Desempregados ou recebendo } \\
\text { benefício }\end{array}$ & 68,8 & 70,5 & 0,659 \\
\hline $\begin{array}{l}\text { Dependem economicamente de } \\
\text { outrem }\end{array}$ & 50,0 & 51,0 & 0,834 \\
\hline Não é arrimo de família & 73,6 & 71,8 & 0,695 \\
\hline $\begin{array}{l}\text { Renda pessoal maior que } \\
1 \text { salário-mínimo }\end{array}$ & 49,7 & 51,1 & 0,785 \\
\hline $\begin{array}{l}\text { Renda familiar de um a três } \\
\text { salários-mínimos }\end{array}$ & 59,9 & 61,3 & 0,809 \\
\hline
\end{tabular}

Nas análises preliminares dos resultados da ACor, foram evidenciadas e selecionadas vinte variáveis, todas com medidas discriminativas superiores a 0,20. Essas 20 variáveis se originaram de apenas três dos 10 domínios abrangidos pela avaliação dos sujeitos: nomeadamente, "identificação de fenômenos reforçadores e/ou desencadeadores do consumo"; "atitude do paciente ante sua problemática" e "delimitação da repercussão macro e microssocial". Ao se analisar as múltiplas correspondências entre os sujeitos, o gráfico de dispersão espacial sugeria, visualmente, uma opção entre dois ou três grupos - com um grupo claramente distinto e dois outros mais próximos entre si -, o que corroborou nossa opção a priori pelos dois clusters.

Os dois clusters identificados pela AC ficaram constituídos por 67 e 236 pacientes, respectivamente. Quando da execução da AC, 26 pacientes foram desconsiderados pelo próprio procedimento estatístico, que excluiu casos com valores missing para qualquer variável utilizada na construção dos clusters. Esses pacientes não foram incluídos nas demais análises.

A Análise Heurística revelou três grupos de variáveis cujos conteúdos semânticos foram categorizados como "comportamentos", "crenças" e "sentimentos": nove referiam-se a crenças relativas a razões para beber ou interromper o con- sumo; nove, a sentimentos que desencadeariam o beber; e duas a comportamentos associados ao uso de alcoólicos.

O quadro 3 expõe as variáveis resultantes da ACor agrupadas pela Análise Heurística e identifica a direção de respostas - afirmativas (admissão de premissa apresentada) ou negativas (não admissão da premissa) - a essas variáveis pelos dois clusters identificados. A maioria dos sujeitos incluídos no cluster 2 respondeu Não a todas as afirmações. Os incluídos no cluster 1 optaram pelo $\underline{\mathrm{Sim}}$ em quase todas essas assertivas: nas três exceções - "Sentir-se abandonado ao começar a beber", "Acreditar que problemas conjugais o levaram a beber muito" e "Acreditar que problemas econômicos o levaram a recair" - os percentuais para a resposta $\underline{\text { Não se mos- }}$ traram bastantes próximos ao limite de inversão para o $\underline{\mathrm{Sim}}$.

Na análise bivariada (Quadro 4), das 51 variáveis clinicamente relevantes ${ }^{1}, 13$ mostraram-se estatisticamente significantes ( $p \leq 0,05)$ e 8 marginalmente significantes $(0,05<$ $\mathrm{p}<0,10$ ) na diferenciação dos clusters (subgrupos).

\section{DISCUSSÃO}

Na análise descritiva da amostra, praticamente não se identificou diferença na comparação entre os alcoolistas que concluíram a avaliação do PADQ e aqueles que não a concluíram e que foram, portanto, excluídos das demais análises. A rigor, deve-se observar uma frequência mínima em tratamento na decisão de se incluir os sujeitos nas análises ${ }^{17}$. Ressalte-se que as duas características que diferenciaram os grupos - naturalidade e média etária - não se mostraram estatisticamente significantes quando da verificação de possíveis associações com os subgrupos identificados ( $p$-valores, respectivamente de 0,504 e 0,317).

Ainda que o presente estudo tenha analisado dados relativos a 10 diferentes domínios na avaliação dos pacientes, as 20 variáveis extraídas pela ACor se originaram de apenas três domínios: "atitude do paciente ante sua problemática", "identificação de fenômenos reforçadores e/ou desencadeadores do consumo" e "delimitação da repercussão macro e microssocial". No processo da Análise Heurística aqui desenvolvida, optou-se por rearticular as variáveis em três grupos de sentido categorizáveis, como comportamentos, crenças e sentimentos.

Ao explicitar que essa análise identificou fatores fundamentalmente pessoais, subjetivos, é relevante sublinhar que está fora de questão que alguns sentimentos possam se originar da interação com o ambiente e que aspectos ambientais influenciem crenças e comportamentos associados ao beber. Ainda que boa parte das pesquisas relacionadas à tipologia de sujeitos dependentes de alcoólicos se concentre na abordagem de aspectos genéticos e neurobiológicos desses sujeitos e nas possibilidades de intervenção farmacológica sobre esse comportamento disfuncional, é reconhecida a relevância dos aspectos psicossociais na caracterização e no tratamento dos diferentes subgrupos de alcoolistas?. 
Quadro 3. Grupos de sentido derivados da análise heurística das variáveis identificadas pela ACor e padrão de respostas dos dois clusters identificados

\begin{tabular}{|c|c|c|c|c|c|c|}
\hline \multirow[t]{2}{*}{ Grupos de sentido } & \multirow[t]{2}{*}{ Variáveis } & \multirow[t]{2}{*}{ Medidas discriminativas } & \multicolumn{2}{|c|}{$\begin{array}{l}\text { Cluster } 1 \\
(\mathrm{n}=67)\end{array}$} & \multicolumn{2}{|c|}{$\begin{array}{l}\text { Cluster2 } \\
(\mathrm{n}=236)\end{array}$} \\
\hline & & & Direção de resposta & $\%$ & Direção de resposta & $\%$ \\
\hline \multirow[t]{9}{*}{ Sentimentos } & Sentir-se incapaz ao começar a beber* & 0,356 & $\operatorname{Sim}$ & 55,2 & Não & 97,0 \\
\hline & Sentir-se perdido ao começar a beber* & 0,336 & Sim & 59,7 & Não & 94,1 \\
\hline & Sentir-se rejeitado ao começar a beber* & 0,294 & $\operatorname{Sim}$ & 55,2 & Não & 92,4 \\
\hline & $\begin{array}{l}\text { Sentir-se sem perspectivas para o futuro a } 0 \\
\text { começar a beber* }\end{array}$ & 0,288 & Sim & 68,7 & Não & 91,9 \\
\hline & $\begin{array}{l}\text { Sentir-se precisando de coragem ao } \\
\text { começar a beber* }\end{array}$ & 0,287 & Sim & 71,6 & Não & 86,9 \\
\hline & $\begin{array}{l}\text { Sentir ter feito algo errado ao começar a } \\
\text { beber* }\end{array}$ & 0,266 & $\operatorname{Sim}$ & 64,2 & Não & 90,3 \\
\hline & Sentir-se carente ao começar a beber* & 0,248 & $\operatorname{Sim}$ & 64,2 & Não & 94,4 \\
\hline & Sentir-se só ao começar a beber* & 0,229 & Sim & 61,2 & Não & 85,2 \\
\hline & Sentir-se abandonado ao começar a beber* & 0,205 & Não & 52,2 & Não & 94,5 \\
\hline \multirow[t]{9}{*}{ Crenças } & Acreditar que ansiedade o levou a recair* & 0,215 & Sim & 53,7 & Não & 89,4 \\
\hline & $\begin{array}{l}\text { Acreditar que problemas econômicos } 0 \\
\text { levaram a recair* }\end{array}$ & 0,252 & Não & 55,2 & Não & 91,1 \\
\hline & Acreditar que depressão o levou a recair* & 0,250 & Sim & 68,7 & Não & 84,3 \\
\hline & $\begin{array}{l}\text { Acreditar que tensão/ansiedade } 0 \text { levaram } \\
\text { a beber muito* }\end{array}$ & 0,252 & Sim & 64,2 & Não & 88,1 \\
\hline & $\begin{array}{l}\text { Acreditar que depressão o levou a beber } \\
\text { muito* }\end{array}$ & 0,243 & $\operatorname{Sim}$ & 83,6 & Não & 77,1 \\
\hline & $\begin{array}{l}\text { Acreditar que problemas econômicos } 0 \\
\text { levaram a beber muito* }\end{array}$ & 0,242 & Sim & 58,8 & Não & 85,6 \\
\hline & $\begin{array}{l}\text { Acreditar que problemas conjugais } 0 \\
\text { levaram a beber muito* }\end{array}$ & 0,217 & Não & 52,2 & Não & 88,6 \\
\hline & $\begin{array}{l}\text { Acreditar que está com problemas } \\
\text { psicológicos** }\end{array}$ & 0,227 & Sim & 67,2 & Não & 80,5 \\
\hline & $\begin{array}{l}\text { Acreditar que evitar problemas econômicos } \\
0 \text { motiva a parar de beber** }\end{array}$ & 0,234 & $\operatorname{Sim}$ & 58,2 & Não & 84,3 \\
\hline \multirow[t]{2}{*}{ Comportamentos } & Envolver-se em agressões ${ }^{* * *}$ & 0,283 & Sim & 85,1 & Não & 52,1 \\
\hline & Beber já causou problemas no trabalho*** & 0,225 & Sim & 64,2 & Não & 74,2 \\
\hline
\end{tabular}

* Domínio "Identificaçăo de fenômenos reforçadores/desencadeadores do consumo"; *** Domínio "Atitude do paciente ante sua problemática"; * *** Domínio "Delimitaçăo da repercussão macro- e micrrossocial".

\section{Análise heurística}

As características dos pacientes incluídos no subgrupo 1 permitem considerá-los como apresentando um consumo de alcoólicos associado à admissão de sentimentos negativos acerca de si mesmos; suas crenças predominantes são de que a tensão/ansiedade e a depressão, bem como aspectos econômicos, são importantes fatores que favoreceriam seu consumo excessivo e sua recaída. Similarmente, apresentaram maior repercussão social associada ao beber: nomeadamente, problemas no trabalho e envolvimento em agressões verbais. Tomadas em conjunto, essas características sugerem maior frequência de problemas associados ao beber ou do beber associado a problemas neste subgrupo. A rigor, não se pode afirmar que os sentimentos e crenças associados a este subgrupo seriam, de fato, reforçadores ou desencadeadores do consumo; todavia, são relevantes para eles e deve-se questionar se uma abordagem psicoterápica que se concentre em sua abordagem não poderia resultar em maior eficácia terapêutica para esse grupo de sujeitos com mais intensa repercussão clínica, conforme discutido mais adiante.

Alcoolistas mais graves possuem pior adaptação familiar, apresentam mais sintomas ansioso-depressivos e tendem a ser impulsivos, o que estaria relacionado com o beber exagerado e dificuldade em se manter abstinente ${ }^{18,19}$. Ainda que fenômenos psicopatológicos possam ser agravados ou aliviados pelo uso de alcoólicos ${ }^{20}$, os resultados aqui encontrados são consistentes com a conhecida relação entre padrões de consumo e características de personalidade ${ }^{21,22}$.

Deve ser ressaltado que o comportamento de consumo dos sujeitos incluídos no subgrupo 1 parece estar mais associado à evitação de sofrimento que a busca de prazer, o 
Quadro 4. Resultados da análise bivariada entre variáveis clinicamente relevantes ${ }^{+}$e os subgrupos identificados

\begin{tabular}{|c|c|c|c|c|}
\hline \multirow[b]{2}{*}{ Variáveis } & \multirow[b]{2}{*}{ p-valor } & \multicolumn{3}{|c|}{ Respostas afirmativas (\%) } \\
\hline & & $\begin{array}{l}\text { Total } \\
(\%)\end{array}$ & $\begin{array}{c}\text { Subgrupo } 1 \\
(\%)\end{array}$ & $\begin{array}{c}\text { Subgrupo } 2 \\
(\%)\end{array}$ \\
\hline Estar em seu $1^{\circ}$ relacionamento conjugal $(n=187)$ & 0,087 & 72,2 & 62,2 & 75,4 \\
\hline Tem filhos ( $n=297)$ & 0,060 & 75,4 & 84,4 & 73,0 \\
\hline Escolaridade até $1^{\circ}$ grau completo $(n=296)$ & 0,008 & 80,1 & 68,7 & 83,4 \\
\hline Renda familiar de até $3 S^{*}(n=255)$ & 0,016 & 59,2 & 45,8 & 63,3 \\
\hline História de alcoolismo paterno $(n=268)$ & 0,090 & 22,0 & 30,4 & 19,8 \\
\hline Faz uso drogas ilícitas ( $\mathrm{n}=301)$ & 0,015 & 14,6 & 23,9 & 12,0 \\
\hline Faz uso de maconha $(n=294)$ & 0,005 & 12,6 & 22,7 & 9,6 \\
\hline Já procurou algum tratamento para 0 alcoolismo $(n=303)$ & 0,084 & 56,4 & 65,7 & 53,8 \\
\hline Beber já causou problema com alguém $(\mathrm{n}=303)$ & 0,016 & 93,7 & 100 & 91,9 \\
\hline Tem problemas sexuais $(\mathrm{n}=300)$ & 0,066 & 80,3 & 72,3 & 82,6 \\
\hline Pontuação superior ao ponto de corte da Escala ? do MMPI** $(n=217)$ & 0,066 & 04,6 & 0,00 & 06,1 \\
\hline Pontuação superior ao ponto de corte da Escala F do MMPI $(n=217)$ & 0,004 & 39,6 & 56,6 & 34,1 \\
\hline Pontuação superior ao ponto de corte da Escala Hs do MMPI $(n=217)$ & 0,064 & 40,1 & 50,9 & 36,6 \\
\hline Pontuação superior ao ponto de corte da Escala D do MMPI $(n=217)$ & 0,054 & 30,9 & 41,5 & 27,4 \\
\hline Pontuação superior ao ponto de corte da Escala Hy do MMPI $(n=217)$ & 0,023 & 22,6 & 34,0 & 18,9 \\
\hline Pontuação superior ao ponto de corte da Escala Pd do MMPI $(n=217)$ & 0,003 & 24,4 & 39,6 & 19,5 \\
\hline Pontuação superior ao ponto de corte da Escala Pa do MMPI $(n=217)$ & 0,001 & 44,7 & 64,2 & 38,4 \\
\hline Pontuação superior ao ponto de corte da Escala Sc do MMPI $(n=217)$ & 0,004 & 39,6 & 56,6 & 34,1 \\
\hline Pontuação superior ao ponto de corte da Escala Si do MMPI $(n=217)$ & 0,006 & 10,6 & 20,8 & 07,3 \\
\hline Pontuação superior ao ponto de corte da Escala MacAndrew $(n=215)$ & 0,017 & 72,1 & 84,9 & 67,9 \\
\hline Pontuação na Escala de MacAndrew acima da média $(26,20)(n=215)$ & 0,019 & 52,1 & 66,0 & 47,5 \\
\hline
\end{tabular}

+ Além das correlaçōes ao menos marginalmente significantes, as outras variáveis utilizadas nas análises bivariadas foram: "idade", "sexo", "raça", "estado civil", "idade em que se casou pela primeira vez", "religião", "frequentar culto religioso", "possuir emprego", "depender economicamente", "ser arrimo de família", "renda pessoal", "apresentou algum problema de saúde, internou-se ou fez tratamento médico no último ano", "fez algum tratamento médico no último ano", "fez uso de medicação no último mês";" "idade que começou a beber"; "apresentou uma ou mais complicaçōes clínicas pelo uso de álcool", "recebeu algum diagnóstico de comorbidade clínica", "apresentou alguma complicação psiquítrica pelo uso de álcool"; "recebeu a algum diagnóstico de comorbidade psiquíátrica"; "subprograma terapêutico ao qual foi encaminhado (farmacológico ou simbólicos)", "tempo de adesão ao tratamento (até 6 meses; superior a 6

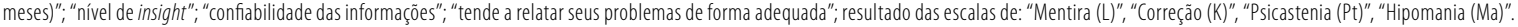

\# Teste do Qui-quadrado de Pearson.

* SM: salário-mínimo; ** Inventário Multifásico Minnesota de Personalidade.

que é coerente com o baixo número de pacientes com características antissociais no grupo aqui estudado - a rigor, nenhum paciente recebeu o diagnóstico de Transtorno de Personalidade Antissocial. Muito embora comportamentos heteroagressivos sejam observados em alcoolistas com sintomatologia depressivo-ansiosa, tal achado ainda careceria de explicação para sua elevada prevalência: segundo Reulbach et al. ${ }^{23}$, tanto efeitos diretos de alcoólicos quanto sua abstinência poderiam provocar as manifestações adrenérgicas associadas a tais comportamentos.

Ainda que o grande percentual de sujeitos incluídos no subgrupo 2 bem como o padrão de respostas dos dois clusters resultante das variáveis identificadas pela ACor e submetidas a uma análise heurística (Quadro 3) - em especial as variáveis em que os dois subgrupos apresentaram a mesma direção de resposta - sugiram a possibilidade de uma solução com maior número de clusters, dados os objetivos deste estudo foi mantida a opção inicial de se restringir as análises a esses dois subgrupos.

\section{Análise bivariada}

Os resultados da análise bivariada indicaram que os pacientes incluídos no subgrupo 1 possuíam melhores níveis de escolaridade e de renda familiar que os do subgrupo 2; também possuíam maior chance de terem filhos e de estarem além do primeiro relacionamento conjugal. Da mesma forma, apresentavam maior percentual de uso de drogas, de problemas na relação com os outros, maior frequência de alterações psicopatológicas - conforme avaliado pelas escalas clínicas e de validade do MMPI ${ }^{15}$-, maiores escores na escala de MacAndrew de alcoolismo e maiores percentuais de relato de procura por tratamento para alcoolismo. Assim, apesar de esses pacientes serem oriundos de estratos socioeconômicos mais favorecidos, os resultados indicam que, ao lado de maior impacto da presença de alcoolismo paterno, eles apresentam um perfil de maior gravidade clínica, em especial consideradas suas características de personalidade.

Ainda que os resultados não tenham sido significantes, pacientes do subgrupo 1 tiveram maior percentual de co- 
morbidade psiquiátrica registrada que os do subgrupo 2 (respectivamente $58,2 \%$ e $47,0 \%, p=0,106$ ). Quando considerados os três grupos de comorbidades mais diagnosticados - Transtornos de Ansiedade, Transtornos de Humor e Transtornos de Personalidade, que respondem por 91,4\% das comorbidades identificadas pelos avaliadores -, observou-se que, apesar da homogeneidade quanto aos Transtornos de Ansiedade (17,9\% e 17,3\%, respectivamente para os subgrupos 1 e 2), os Transtornos de Humor predominaram entre os pacientes do subgrupo 1 (48,7\% contra 28,6\%) e os Transtornos de Personalidade, no subgrupo 2 (33,3\% e $54,1 \%), p=0,055$. Tais resultados reforçam a possibilidade de que os sentimentos e crenças associados de forma relevante ao subgrupo 1 não tenham relação direta com os diagnósticos psiquiátricos, ainda que se associem ao consumo e possam ser eficazmente trabalhados por meio de abordagem psicoterápica específica.

Além do tabaco, utilizado por $74 \%$ dos sujeitos, sem diferença entre os subgrupos ( $p=0,272)$, apenas o uso de maconha $(12,6 \%)$ e cocaína $(8,2 \%)$ ultrapassou o limite de $2 \%$, sempre mais elevado - e de forma estatisticamente significante no caso da maconha $(p=0,005)$ - entre os indivíduos do subgrupo 1; tais achados estão de acordo com os resultados de Wittchen et al..24, que observaram que alcoolistas com maior frequência de relato de uso de maconha possuíam maior probabilidade de comorbidades psiquiátricas.

A tipologia de Babor identificou dois grupos de alcoolistas, descritos como Tipo A e Tipo B. Os alcoolistas do Tipo A foram caracterizados como sendo mais conservadores - isto é, menos propensos a experiências e novidades - e seus problemas ligados ao álcool teriam início mais tardio. Também apresentariam menor grau de dependência, menores taxas de consequências físicas e sociais relacionadas ao consumo alcoólico, menores taxas de comorbidade psiquiátricas e de alterações relacionadas ao trabalho e à família, bem como progressão mais lenta do quadro e melhor prognóstico. Os do Tipo B foram considerados mais experimentadores e mais tensos; apresentariam ainda: início mais precoce dos problemas ligados ao álcool; maior grau de dependência; maiores taxas de abuso de outras substâncias; consequências físicas e sociais mais sérias; maior frequência de comorbidade psicopatológica; progressão mais rápida do alcoolismo e pior prognóstico ${ }^{3-6}$.

Por sua vez, a tipologia de Cloninger et al. identificou um grupo de sujeitos, o Tipo 2, caracterizado por início mais precoce dos problemas relacionados ao álcool e maior envolvimento em brigas e prisões. Alcoolistas do Tipo 2 tenderiam mais à busca de novidades, à impulsividade e a apresentarem ansiedade somática e características antissociais e a terem na indução de euforia forte motivação para o beber. Alcoolismo grave e criminalidade do pai biológico seriam fatores relevantes nesse subtipo ${ }^{25}$. Em relação aos traços de personalidade, alcoolistas do Tipo 1 apresentariam frequência mais elevada de ansiedade cognitiva, de comportamentos de evitação de danos e de busca de gratificação e de consumo para alívio de ansiedade ${ }^{20}$.

Assim, o subgrupo 1 aqui identificado se aproxima, por suas características de personalidade, do Tipo A de Babor e do Tipo 1 de Cloninger, ao passo que, pela gravidade clínica, se assemelha ao Tipo B de Babor e ao Tipo 2 de Cloninger. Sabe-se que tipologias desenvolvidas a partir de amostras de pacientes em tratamento - ou hospitalizados (como a de Babor) - implicam um viés de seleção pela inclusão de casos de maior repercussão clínica e/ou gravidade. Subtipos assim identificados não são adequados para triagem, identificação precoce ou prevenção de alcoolismo ${ }^{17}$, como seria o caso de tipologias derivadas de amostras comunitárias ${ }^{26}$. A tipologia de Cloninger, apesar de não se utilizar de uma amostra comunitária típica, tampouco partiu de sujeitos em tratamento. Os pacientes aqui estudados representam a realidade clínica de nosso meio: são pacientes com grave síndrome de dependência alcoólica sem que, contudo, apresentem o nível de impulsividade e de busca de gratificação dos sujeitos incluídos nos estudos de Babor e Cloninger. Apesar de o subgrupo 1 ter apresentado características de maior gravidade clínica, não manifestaram as especificidades genéticas e de personalidade do Tipo 2 de Cloninger, o que sugere que sua história de alcoolismo tenha sofrido mais a influência de fatores ambientais que genéticos.

Ressalta-se que os subprogramas terapêuticos aos quais os pacientes foram encaminhados não se associaram ao tempo de adesão ao tratamento em cada subgrupo, tampouco foram estatisticamente significantes na diferenciação dos subgrupos aqui identificados, sugerindo que o PADQ teria similarmente atendido às necessidades desses subgrupos de pacientes. Ainda assim, é possível supor que novas abordagens diagnósticas e terapêuticas adequadas às necessidades especíicas de cada um dos subgrupos - isto é, abordagens que enfoquem as distorções cognitivas, afetivas e comportamentais desses sujeitos - possam contribuir para tornar mais eficaz e efetivo o atendimento ambulatorial de alcoolistas em nosso meio ${ }^{27}$.

\section{Limitações}

Uma vez que a admissão dos sujeitos aqui estudados obedeceu tão somente a considerações clínico-administrativas que, a rigor, excluíam a possibilidade de referência ao serviço de pacientes com o diagnóstico, teoricamente menos grave, de uso nocivo -, deve-se atentar para a reduzida possibilidade de generalização dos resultados do presente estudo, uma das limitações deste como de todos os estudos observacionais. Outra limitação decorre do fato de que os dados aqui utilizados advêm, em parte, de instrumentos que coletavam dados relativos a sintomas e padrões de consumo pregressos, o que pode resultar em viés de memória. 
Todavia, estudos que se desdobram a partir de circunstâncias de "vida real" têm sido cada vez mais valorizados, exatamente por possibilitarem importantes discussões acerca de aspectos relativos à efetividade das práticas assistenciais $^{28,29}$. O elevado número de pacientes incluídos raramente é encontrado em estudos brasileiros e sua simplicidade de desenho permite que possa ser facilmente reproduzido em diferentes contextos assistenciais.

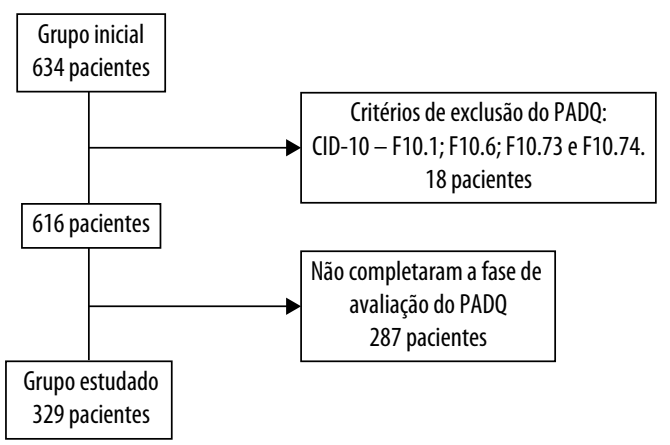

PADQ: serviço ambulatorial de referência para alcoolistas; CID-10: Classificação Internacional de Doenças, $10^{a}$ edição.

Figura 1. Fluxograma de inclusão e exclusão.

\section{CONCLUSÕES}

Os resultados deste estudo permitem supor que a tipologia aqui apresentada - resultante da articulação de ferramentas estatísticas e pressupostos teóricos - possa contribuir para a discussão sobre a relevância clínica da abordagem tipológica de alcoolistas. Além de indicar a factibilidade de construção, em nosso meio, de uma tipologia binária que permita estudos comparativos com outras tipologias binárias, é possível supor que ela possa ser efetivamente aplicada, em nosso meio, em serviços de atendimento a alcoolistas que não se concentrem na pesquisa. Em especial, é possível supor que essa tipologia possa contribuir para o tratamento desses dois subtipos de alcoolistas, por meio de uma estratégia teórico-prática adaptada às necessidades desses sujeitos, o que seria o objeto de futuros estudos.

\section{CONTRIBUIÇÕES INDIVIDUAIS}

Mário Sérgio Ribeiro - Coordenador do estudo, participou de todas as suas fases, incluindo na análise dos dados e redação do artigo.

Luiz Cláudio Ribeiro - Responsável pelas análises estatísticas e orientação da apresentação dos resultados.

Rafael Augusto Ferreira - Responsável pela revisão bibliográfica, tratamento e interpretação dos dados e redação inicial do artigo.

\section{CONFLITOS DE INTERESSE}

Os autores declaram não haver conflitos de interesse.

\section{AGRADECIMENTOS}

Os autores agradecem à Universidade Federal de Juiz de Fora (UFJF), ao Conselho Nacional de Desenvolvimento Científico e Tecnológico (CNPq) e ao Departamento de Saúde Mental (DSM) da Prefeitura Municipal de Juiz de Fora pelas bolsas de Iniciação Científica concedidas a estudantes que, em diferentes momentos, participaram da coleta de dados do estudo observacional de alcoolistas ou das análises que resultaram neste artigo.

\section{REFERÊNCIAS}

1. Donovan DM. Assessment to aid in the treatment planning process. In: Allen JP, Wilson VV. Assessing alcohol problems: a guide for clinicians and researchers. 2.ed. Bethesda: US Department do Health and Human Services/National Institute on Alcohol Abuse and Alcoholism; 2003. p. 125-88.

2. Ribeiro MS, Ribeiro LC, Garcia MA, Souza GF, Oliveira LN. Avaliação dos tipos 1 e 2 de alcoolismo de Cloninger em homens participantes de um programa de tratamento ambulatorial. Rev Psiquiatr Clín. 2008;35(2):39-48.

3. Ribeiro MS, Ribeiro LC, Garcia MA, Souza GF, Nogueira RB, Sousa KDC. Aplicabilidade da classificação de alcoolismo tipo A/tipo B. J Bras Psiq. 2009;58(1):17-25.

4. Babor TF, Hofmann M, DelBoca FK, Hesselbrock V, Meyer RE, Dolinsky, et al. Types of alcoholics, I: evidence for an empirically derived typology based on indicators of vulnerability and severity. Arch Gen Psychiatry. 1992;49:599-608.

5. Ribeiro MS, Guirro UBP, Baldi BG. Tipologias em alcoolismo: diagnóstico e terapêutica. Arq Bras Psiquiatr Neurol Med Legal. 2002:80/81:24-32.

6. Brown J, Babor TF, Litt MD, Kranzler HR. The type A/type B distinction: subtyping alcoholics according to indicators of vulnerability and severity. Ann NY Acad Sci. 1994;708:23-33.

7. Babor TF, Caetano R. Subtypes of substance dependence and abuse: implications for diagnostic classification and empirical research. Addiction. 2006;101(1):104-10.

8. Leggio L, Kenna GA, Fenton M, Bonenfant E, Swift Rm. Typologies of alcohol dependence from Jellinek to genetics and beyond. Neuropsychol Rev. 2009;19:115-29.

9. Hillemacher T, Bleich S. Neurobiology and treatment in alcoholism - Recent findings regarding. Alcohol Alcohol. 2008;43(3):341-6.

10. Kogoj D, Lesch OM, BlümI V, Riegler A, Vyssoki B, SchlaffG, et al. Lesch alcoholism typology medical treatment and research. Arch Psychiatr Psychother. 2010;4:37-48.

11. Hesselbrock VM, Hesselbrock MN. Are there empirically supported and clinically useful subtypes of alcohol dependence? Addiction. 2006;101(1):97-103.

12. Del Boca FK. Two subtypes or more, much work remains: a commentary on Windle and Scheidt. Addiction. 2004;99(12):1609-10.

13. Windlem, Scheidt dm. Alcoholic subtypes: are two sufficient? Addiction. 2004;99(12):150819.

14. Benkö A, Simões RJP. Inventário multifásico Minesota de personalidade - Manual (tradução e adaptação). Rio de Janeiro: (EPA; s/d.

15. Batista LE, Escuder MML, Pereira JCR. A cor da morte: causas de óbito segundo características de raça no Estado de São Paulo, 1999 a 2001. Rev Saúde Pública. 2004;38(5):630-6.

16. Pachur T, Todd PM, Gigerenzer G, Schooler LJ, Goldstein DG. The recognition heuristic: a review of theory and tests. Frontiers in Psychology: Cognitive Science. 2011;2:1-14.

17. Holder H. Prevention programs in the 21st century: what we do not discuss in public. Addiction. 2010;105(4):578-81. 
18. Echeburúa E, Medina RB, Aizpiri J. Alcoholism and personality disorders: an exploratory study. Alcohol Alcohol. 2005;40(4):323-6.

19. Marmorstein NR. Longitudinal associations between alcohol problems and depressive symptoms: early adolescence through early adulthood. Alcohol Clin Exp Res. 2009;33(1):49-59.

20. Driessen M, Veltrup C, Wetterling T, John U, Dilling H. Axis I and Axis II comordity in alcohol dependence and the two types of alcoholism. Alcohol Clin Exp Res. 1998;22(1):77-86.

21. MacAndrew C. What the MAC tells us about men al coholics: an interpretative review. Quart J Stud Alc. 1981;42:604-25.

22. Wolf AW, Schubert DSP, Patterson M, Grande T, Pendleton L. The use of the MacAndrew scale in detecting substance abuse and antisocial personality. J Pers Assess. 1990;54(3/4):747-55

23. Reulbach U, Biermann T, Bleich S, Hillemacher T, Kornhuber J, Sperling W. Alcoholism and homicide with respect to the classification systems of Lesch and Cloninger. Alcohol Alcohol. 2007;42(2):103-7.
24. Wittchen HU, Behrendt S, Höfler M, Perkonigg A, RehmJ, Lieb R, et al. A typology of cannabis-related problems among individuals with repeated illegal drug use in the first three decades of life: evidence for heterogeneity and different treatment needs. Drug Alcohol Depend. 2009;102:151-7.

25. Cloninger CR, Bohman M, Sigvardsson S. Inheritance of alcohol abuse. Arch Gen Psychiatry. 1981;38:861-8.

26. Moss $H B$, Chen $C M$, Yi H. Subtypes of alcohol dependence in a nationally representative sample. Drug Alcohol Depend. 2007;91:149-58.

27. Ribeiro MS, Ferreira RA, Souza GF, Ribeiro LC. Características de alcoolistas associadas à adesão prolongada ao tratamento em um programa ambulatorial. Acta Med Port. 2010;23:965-72.

28. Corrao G, Bagnardi V, Zambon A, Aricò S, Dall'Aglio C, Addolorato G. Outcome variables in the evaluation of alcoholics' treatment: Iessons from the Italian Assessment of Alcoholism Treatment (ASSALT) Project. Alcohol Alcohol. 1999;34(6):873-81.

29. Haro JM, Suarez D, Novick D, Usall J, Naber D. Three-year antipsychotic effectiveness in the outpatient care of schizophrenia: observational versus randomized studies results. Eur Neuropsychopharmacol. 2006;:235-44. 